\title{
OPTIMAL POLARIMETRIC DETECTION FILTER AND ITS STATISTICAL TESTS FOR A SHIP DETECTOR
}

\author{
Tao Liu ${ }^{1,2}$, Ricardo Y. C. L. Dias ${ }^{1}$, Jian Yang ${ }^{2}$, Armando Marino ${ }^{3}$, Gui Gao $^{4}$ \\ 1 Naval University of Engineering 2 Tsinghua University 3 the University of Stirling 4 Southwest \\ Jiaotong University
}

\begin{abstract}
Ship detection is one important task in radar remote sensing. Moreover, Polarimetry shows a valuable contribution to discriminate between targets and clutter. The performance of most polarimetric detectors depends on two important factors: target clutter ratio (TCR) and speckles (or standard deviation to mean ratio of clutter background). The polarimetric matched filter (PMF) is just to maximize the TCR, while the polarimetric whitening filter (PWF) only takes the speckle reduction into consideration. In this paper, the optimal polarimetric detection filter (OPDF) is put forward, which considers maximizing the ratio of TCR to speckle. The approximate expression of the probability density function (PDF) of the OPDF is derived in closed form, so are the probability of false alarm (PFA) and the probability of detection (PD) in Wishart distribution assumption. The threshold of the OPDF detection can be easily obtained in closed form or via the bisection method. Experiments via simulated data validate the correctness of our results. The OPDF detector gives the best performance in most environments, especially in low PFA case and in the case where the statistics of targets is not the ideal Wishart distribution.

Index Terms-Polarimetric detection, Synthetic Aperture Radar, Target to Clutter Ratio, Speckle, Optimal Polarimetric Detection
\end{abstract}

\section{INTRODUCTION}

Synthetic aperture radar (SAR) is a useful tool to detect the interesting targets over the ocean. SAR has the ability to monitor ships at nighttime, under cloud cover, and with resolution in meters. Therefore, SAR was widely used in recent years to monitor ships from satellites [1]. It is possible to separate vessels from the background by performing a statistical test on the intensity of the sea clutter for the backscattering signal from ships is usually larger than that from sea. When the probability density function (PDF) of the clutter intensity is known, the problem of selecting the detector threshold can be solved using the Neyman-Pearson (NP) lemma on the probability of detection (PD) or false alarms (PFA) [2]. Considering the benefits of polarimetry in ship detection, several polarimetric detectors were proposed in the recent years. The optimal polarimetric detection (OPD) gave the best performance [3] in theory. Since the target to clutter ratio (TCR) is a quite important factor in target detection, the polarimetric matched filter (PMF) and the optimal polarimetric contrast enhancement (OPCE) were raised to improve the detection performance [3][4]. Generally, the statistical distribution of ships is difficult to obtain; therefore, the tests are generally based only on PFA. In particular, the threshold is selected in order to keep PFA constant [i.e., constant false-alarm rate (CFAR)] [5]. The polarimetric whitening filter (PWF) was proposed for target detection, which minimizes the speckle (or the standard deviation to mean ratio) [6]. The PWF detector achieves a comparable performance with the OPD [6]. Recently the Polarimetric Notch Filter (PNF) was put forward [7] based on some rationale linked to the physical behavior of the sea clutter. The statistical analysis was presented [1].

From the above methods it can be seen that the TCR and speckles are important for target detection in PolSAR imagery. The performance of a detector is always decided by both the important factors. The PWF gives us a minimized fluctuation of speckles. The PMF and the OPCE are aimed to obtain a maximum TCR. It is wondering if there exists a method to combine both the factors together to get a new detector with a better performance. If we process the image with the PWF first, following by the PMF method, in fact it is just the same as the PMF method. Therefore how to combine both the factors is a big problem in target detection to improve the detection performance.

The problem is solved in this paper, and a new detector named optimal polarimetric detection filter (OPDF) is proposed. The performance of the OPDF is studied at the same time. Experiments demonstrate that the OPDF detector gives a comparable performance with the OPD detector. Generally the OPDF detector is better than the existing PMF, PWF and PNF (Notch) detectors.

\section{OPTIMAL POLARIMETRIC DETECTION FILTER}

In the reciprocal assumption, the polarimetric scattering vector, which involves the full polarization information of

(C) 2019 IEEE. Personal use of this material is permitted. Permission from IEEE must be obtained for all other uses, in any current or future media, including reprinting/republishing this material for advertising or promotional purposes, creating new collective works, for resale or redistribution to servers or lists, or reuse of any copyrighted component of this work in other works. 
targets in a determined condition, can be represented on the Horizontal-Vertical basis as [8] ;

$$
\mathbf{s}=\left[\begin{array}{c}
S_{h h} \\
\sqrt{2} S_{h v} \\
S_{v v}
\end{array}\right]
$$

where $S_{x y}$ represents the complex scattering coefficient with $x$ as the transmitting, and $y$ as the receiving polarization (h-horizontal, v-vertical). $\sqrt{2}$ is added to keep the total energy the same.

To suppress the speckle in the SAR imagery, the multilook is always applied among neighboring pixels. In the multi-look case, the covariance matrix describes the full polarization information and it's defined as [8]:

$$
\mathbf{C}=\frac{1}{L} \sum_{i=1}^{L} \mathbf{s}_{i} \mathbf{s}_{i}^{H}
$$

where $L$ is the nominal number of looks, $\boldsymbol{\Sigma}=\mathrm{E}\left\{\mathbf{s} \mathbf{s}^{H}\right\}$ is the expectation of the covariance matrix, C.E $(\bullet)$ denotes the expectation operator.

The essence of the PWF is a linear transform of the three polarization channels (HH HV VV)[6], turning the scattering vector to another space. We follow this thought to find a new transform matrix $\mathbf{G}^{1 / 2}$, which combines the TCR and speckle together to improve the detection performance. That is:

$$
\mathbf{y}=\mathbf{G}^{1 / 2} \mathbf{s}
$$

Then the intensity of the vector $\mathbf{y}$ can be expressed as:

$$
z=\mathbf{y}^{H} \mathbf{y}=\left(\mathbf{G}^{1 / 2} \mathbf{s}\right)^{H} \mathbf{G}^{1 / 2} \mathbf{s}=\mathbf{s}^{H} \mathbf{G} \mathbf{s}
$$

In the multi-look case, the intensity $z$ can be expressed as:

$$
z=\frac{1}{L} \sum_{i=1}^{L} \mathbf{s}_{i}{ }^{H} \mathbf{G} \mathbf{s}_{i}=\operatorname{tr}(\mathbf{G C})
$$

The TCR can be defined as:

$$
T C R=\frac{E\left(z_{T}\right)}{E\left(z_{C}\right)}
$$

The ratio of the standard of the image pixel intensity to the mean of the intensities is [6]:

$$
\frac{s}{m}=\frac{\text { st.dev. }\left(z_{C}\right)}{\mathrm{E}\left(z_{C}\right)}=\frac{\sqrt{\operatorname{Var}\left(z_{C}\right)}}{\mathrm{E}\left(z_{C}\right)}
$$

Since the performance of the detection needs high TCR and low fluctuation, we define a new variable to detect targets:

$$
M=\frac{T C R}{(s / m)}
$$

We should maximize the variable $M$ to obtain the best performance of the detection, and then we get:

$$
M^{2}=\left[\frac{T C R}{(s / m)}\right]^{2}=\left[\frac{\mathrm{E}\left(z_{T}\right)}{\mathrm{E}\left(z_{C}\right)} / \frac{\sqrt{\operatorname{Var}\left(z_{C}\right)}}{\mathrm{E}\left(z_{C}\right)}\right]^{2}=\frac{\mathrm{E}^{2}\left(z_{T}\right)}{\operatorname{Var}\left(z_{C}\right)}
$$

We can rewrite the above equation as:

$$
M^{2}=\frac{\mathrm{E}^{2}\left(z_{T}\right)}{\operatorname{Var}\left(z_{C}\right)}=\frac{\operatorname{tr}^{2}\left(\boldsymbol{\Sigma}_{T} \mathbf{G}\right)}{\operatorname{tr}\left(\boldsymbol{\Sigma}_{C} \mathbf{G}\right)^{2}}
$$

We denote $\mathbf{A}=\boldsymbol{\Sigma}_{T} \mathbf{G}, \mathbf{B}=\boldsymbol{\Sigma}_{C} \mathbf{G}$. Via the trace equation $\operatorname{tr}^{2}(\mathbf{A B}) \leq \operatorname{tr}\left(\mathbf{A}^{2}\right) \operatorname{tr}\left(\mathbf{B}^{2}\right)$ [9]. When the equality is built, it should be satisfied that $\mathbf{A}=\mathbf{B}$.

Then:

$$
M^{2}=\frac{\operatorname{tr}^{2}(\mathbf{A})}{\operatorname{tr}\left(\mathbf{B}^{2}\right)}=\frac{\operatorname{tr}^{2}\left(\mathbf{A} \mathbf{B}^{-1} \mathbf{B}\right)}{\operatorname{tr}\left(\mathbf{B}^{2}\right)} \leq \operatorname{tr}\left(\mathbf{A B} \mathbf{B}^{-1}\right)^{2}=\operatorname{tr}\left(\boldsymbol{\Sigma}_{T} \boldsymbol{\Sigma}_{C}^{-1}\right)^{2}
$$

When the equality is satisfied, we can get:

$$
\boldsymbol{\Sigma}_{T} \mathbf{G}\left(\boldsymbol{\Sigma}_{C} \mathbf{G}\right)^{-1}=\boldsymbol{\Sigma}_{C} \mathbf{G}
$$

The simplification is:

$$
\mathbf{G}=\boldsymbol{\Sigma}_{C}^{-1} \boldsymbol{\Sigma}_{T} \boldsymbol{\Sigma}_{C}^{-1}
$$

Then we have derived the transform matrix of the optimal polarimetric detection filter (OPDF) $\mathbf{G}=\boldsymbol{\Sigma}_{C}^{-1} \boldsymbol{\Sigma}_{T} \boldsymbol{\Sigma}_{C}^{-1}$.

When the target information is unknown, we could use the maximum like-hood estimation (MLE) to replace the target covariance matrix. Then the transform matrix turns to be $\mathbf{G}=\boldsymbol{\Sigma}_{C}^{-1}$, that is just the transform matrix of PWF.

\section{STATISTICS OF THE NOVEL DETECTORS}

Many distributions of detection variables turn to be a quadratic form. There is no analytical expression in closeform for a quadratic form [10]. There are many numerical calculation methods to solve this problem. The imhof's method can give the exact result while the calculation is complicated [10]. There are two approximations proposed to simplify the calculation of the distribution of quadratic forms [11]. We can extend the results to the complex case via the method in literature [12]. The extended theorem is as follows:

Theorem 1. Let $\mathbf{x} \sim N_{d}(0, \boldsymbol{\Sigma})$ be a d-variate Gaussian random variable with complex positive definite Hermitian covariance matrix $\mathbf{\Sigma}$, and $z=\mathbf{x}^{H} \mathbf{W} \mathbf{x}$ is a quadratic form in $\mathbf{x}$. The matrix $\mathbf{W}$ is nonnegative definite. Let the rank of $\mathbf{W}$ be $d$ and the nonzero eigenvalues of $\mathbf{W} \mathbf{\Sigma}$ be $\lambda_{1}, \lambda_{2}, \ldots$, $\lambda_{d}$. The approximating distribution of $T$ can be

$$
z \sim c \chi_{2 d}^{2} / 2 \text { or } T \sim a \chi_{2 b}^{2} / 2
$$

where $c=\sum_{i=1}^{d} \lambda_{i} / d, a=\frac{\sum_{i=1}^{d} \lambda_{i}^{2}}{\sum_{i=1}^{d} \lambda_{i}}, \quad b=\frac{\left(\sum_{i=1}^{d} \lambda_{i}\right)^{2}}{\sum_{i=1}^{d} \lambda_{i}^{2}}$ and $H$ is the supper script of conjugate transpose. The related parameters can be estimated as [11]: 


$$
\begin{aligned}
& \hat{c}=\operatorname{tr}(\hat{\mathbf{W}} \hat{\boldsymbol{\Sigma}}) / d \quad, \quad \hat{a}=\operatorname{tr}\left[(\hat{\mathbf{W}} \hat{\boldsymbol{\Sigma}})^{2}\right] / \operatorname{tr}(\hat{\mathbf{W}} \hat{\boldsymbol{\Sigma}}) \quad \text { and } \\
& b=[\operatorname{tr}(\hat{\mathbf{W}} \hat{\boldsymbol{\Sigma}})]^{2} / \operatorname{tr}\left[(\hat{\mathbf{W}} \hat{\boldsymbol{\Sigma}})^{2}\right] .
\end{aligned}
$$

The above theorem is important to our derivations. $z \sim a \chi_{2 b}^{2} / 2$ is equivalent to $z \sim \gamma(b, a)$. Here $\gamma(\alpha, \beta)$ is the gamma distribution, which can be expressed as follows[13]:

$$
p_{\gamma}(\alpha, \beta)=\frac{1}{\Gamma(\alpha)} \frac{1}{\beta}\left(\frac{x}{\beta}\right)^{\alpha-1} e^{-\frac{x}{\beta}}
$$

$\alpha$ is the shape parameter, and $\beta$ is the scale parameter.

In multilook case, the sum of the independent gamma distributed variables is distributed as follows according to the characteristics of gamma distribution [13]:

$$
z_{S}=\sum_{i=1}^{L} T_{i} \sim \gamma(L b, a)
$$

where $L$ is the equivalent number of looks.

Therefore according to the characteristics of gamma distribution [13] the distribution of the average of the sum is:

$$
z_{L}=z_{S} / L \sim \gamma\left(L b, \frac{a}{L}\right)
$$

If the detection threshold is assumed to be $T$, the PFA or PD can be determined as:

$$
P_{f a / d}=\int_{T}^{\infty} \gamma\left(L b, \frac{a}{L}\right) d z=\frac{\Gamma\left[L b, \frac{L T}{a}\right]}{\Gamma[L b]}
$$

where

$$
\Gamma(x)=\int_{0}^{\infty} e^{-t} t^{x-1} d t, \Gamma(a, x)=\int_{x}^{\infty} e^{-t} t^{a-1} d t
$$

$P_{f a}$ is the PFA, $P_{d}$ is the PD, and $L$ is the number of looks. Therefore, the threshold $T$ can be derived as:

$$
T=\frac{\Gamma^{-1}\left[L b, P_{f a} \Gamma[L b]\right] a}{L}
$$

where $\Gamma^{-1}[\cdot]$ is the inverse incomplete Gamma function [13]. In the OPDF detector, $\mathbf{W}=\mathbf{G}=\boldsymbol{\Sigma}_{C}^{-1} \boldsymbol{\Sigma}_{T} \boldsymbol{\Sigma}_{C}^{-1}$. The difference between the $P_{f a}$ and $P_{d}$ lies in the different choice of the covariance matrix $\boldsymbol{\Sigma}\left(\boldsymbol{\Sigma}_{T}\right.$ or $\left.\boldsymbol{\Sigma}_{C}\right)$ in the definition of $a$ and $b$. So does the approximation with $c$ and $d$.

We should note that when $\mathbf{G} \boldsymbol{\Sigma}=\mathbf{I}$, the approximation will be exact, where I is an identity matrix[14].

\section{EXPERIMENTS AND VALIDATION}

We will validate these approximations to simplify the calculation of the OPDF detector. Then we analyze the detection performance of the OPDF detector. The simulated data including clutter and targets are both generated from the real data (a 9-look NASA/JPL AIRSAR polarimetric dataset that covers an area of the inland sea named Kojimawan close to Tamano City, Japan, October 4, 2000). Since target is much stronger than sea clutter generally, we change TCR to study the detection performance of small targets, where $T C R=\operatorname{tr}\left(\Sigma_{T}-\Sigma_{C}\right) / \operatorname{tr}\left(\Sigma_{C}\right)$ [3].

\subsection{Goodness of fit of the approximations}

The comparisons among the simulated results, imhof's method, approximation with ab, and approximation with cd are presented. From Fig. 1 and Fig. 2 it can be seen that the imhof's method gives the exact result, and the approximation with ab obtains better goodness fit than that of cd. The results are in accordance with that in [11]. And the approximations of PFA are more accurate than PD.

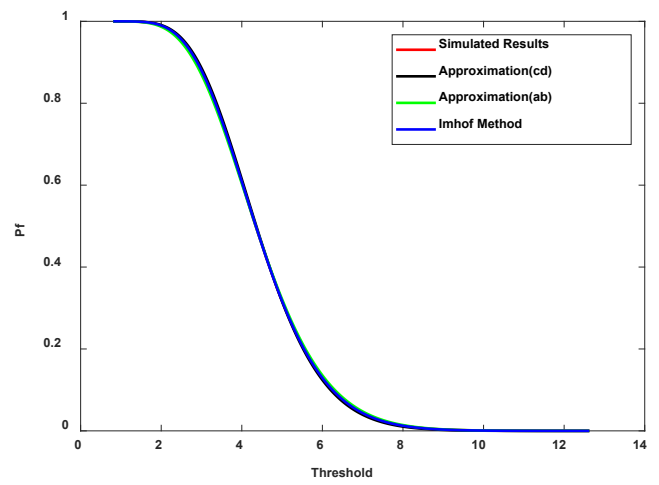

Fig 1. Comparisons among the four calculations of PFA

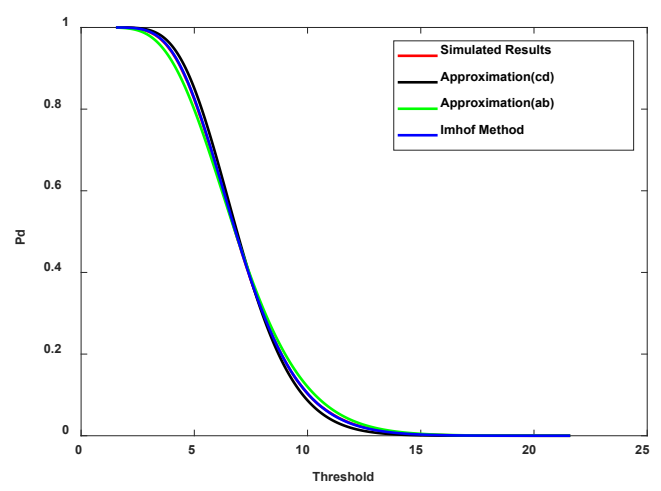

Fig 2. Comparisons among the four calculations of PD

\subsection{Performance of polarimetric detectors}

The performances of most existing polarimetric detectors are presented in Fig 3 and Fig 4. The curves including receiver operating characteristic (ROC) show the performance of the OPDF is comparable with that of OPD, and better than all others.

\subsection{Adaptability to the statistical models of targets}

Though clutter is often Wishart distributed, targets usually do not obey this distribution. They may obey the KWishart distribution or G0-Wishart distribution actually. Fig 5 and Fig 6 give the detection performances in different target models. It shows that the OPDF gives the best performance in all classical polarimetric detectors. 


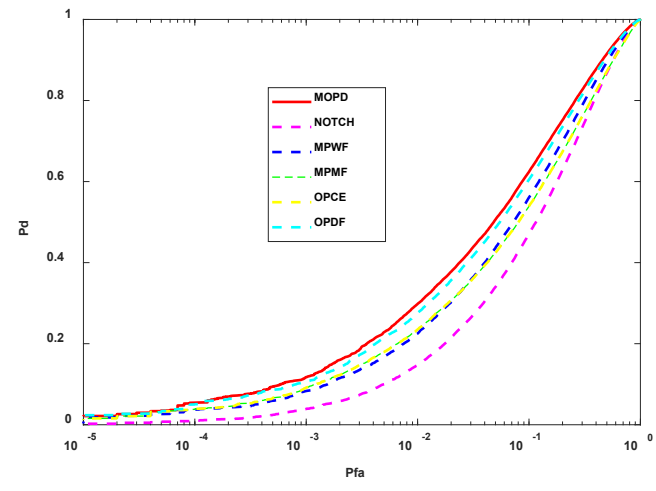

Fig 3. The ROC curves with $\mathrm{TCR}=0.5$

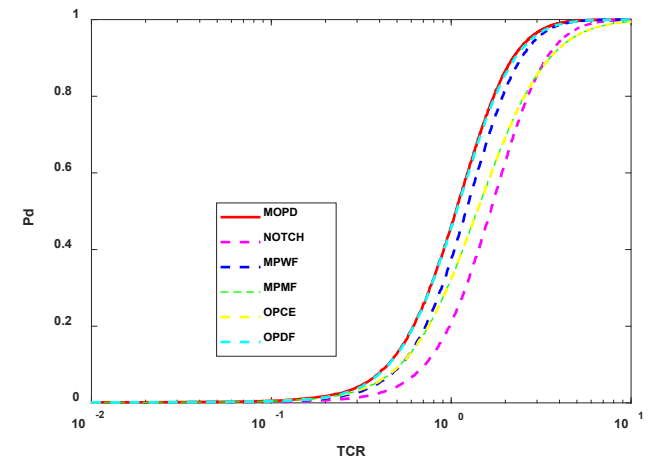

Fig 4. The PD varying with the TCR when $P F A=1 e-3$

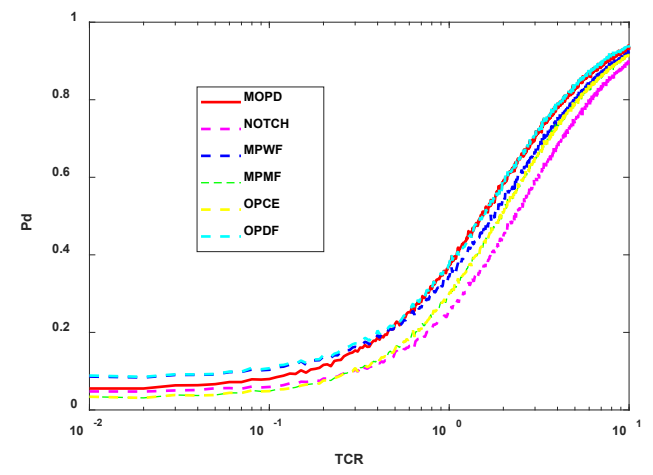

Fig 5. The PD varying with the TCR when PFA=1e-3 in KWishart case

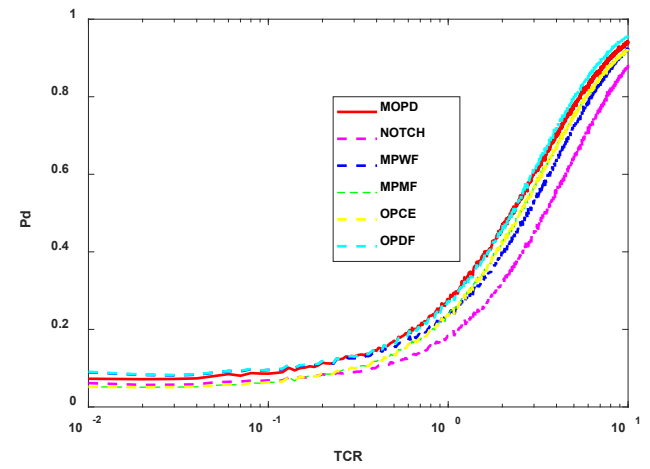

Fig 6. The PD varying with the TCR when $P F A=1 e-3$ in G0Wishart case

\section{CONCLUSION}

A novel polarimetric detector named OPDF was proposed in this paper. The OPDF detector gave the best performance in most environments, especially in low PFA case and when the statistical model of targets is not the Wishart distribution.

\section{ACKNOWLEDGMENT}

This work was supported by the National Natural Science Foundation of China (61771483; 61490693; 61771043).

\section{REFERENCES}

[1] A. Marino and I. Hajnsek, "Statistical Tests for a Ship Detector Based on the Polarimetric Notch Filter," IEEE Transactions on Geoscience and Remote Sensing, vol. 53, no. 8, pp. 4578-4595, Aug. 2015.

[2] S. M. Kay, Fundamentals of Statistical Signal Processing, Upper Saddle River, NJ, USA: Prentice Hall, 1993.

[3] L. M. Novak, M. B. Sechtin and M. J. Cardullo, "Studies of target detection algorithms that use polarimetric radar data," IEEE Transactions on Aerospace and Electronic Systems, vol. 25, no. 2, pp. 150-165, March 1989.

[4] J. Yang, Y. Yamaguchi, W.-M. Boerner, and S. M. Lin, "Numerical methods for solving the optimal problem of contrast enhancement," IEEE Trans. Geosci. Remote Sensing, vol. 38, pp. 965-971, Mar. 2000.

[5] G. Gao and G. Shi, "CFAR Ship Detection in Nonhomogeneous Sea Clutter Using Polarimetric SAR Data Based on the Notch Filter," IEEE Transactions on Geoscience and Remote Sensing, vol. 55, no. 8, pp. 4811-4824, Aug. 2017. [6] Novak L M, Burl M C, " Optimal speckle reduction in polarimetric SAR imagery," IEEE Transactions on Aerospace and Electronic Systems, vol.26, no. 2, pp. 293-305, 1990.

[7] A. Marino, S. R. Cloude and I. H. Woodhouse, "Detecting Depolarized Targets Using a New Geometrical Perturbation Filter," IEEE Transactions on Geoscience and Remote Sensing, vol. 50, no. 10, pp. 3787-3799, Oct. 2012.

[8] Liu Tao, Cui Haogui, Xi Zemin, Gao Jun, “ Novel Estimators of Equivalent Number of looks in Polarimetric SAR imagery based on Sub-matrices," Science China Information Science, vol. 59, no. 6, pp.1869-1919, 2016.

[9] X. Yang, X. Yang, and K. L. Teo, "A matrix trace inequality," J. Math.Anal. Appl., vol. 263, pp. 327-333, 2001.

[10] J P Imhof, "Computing the distribution of quadratic forms in normal variables,” Biometrika, 48(3/4), pp:419-426 ,1961.

[11] Yuan K-H, Bentler PM, "Two Simple Approximations to the Distributions of Quadratic Forms," The British journal of mathematical and statistical psychology. 63(Pt 2):273-291. [12] N. Giri, "On the complex analogues of T2- and R2-tests," Ann. Math.Stat., vol. 36, no. 2, pp. 664-670, Apr. 1965.

[13] C. Forbes, M. Evans, N. Hastings, and B. Peacock, Statistical Distributions, 4th ed. Hoboken, NJ, USA: Wiley, 2010.

[14] Khan S, Guida R, “On Fractional Moments of Multilook Polarimetric Whitening Filter for Polarimetric SAR Data," IEEE Transactions on Geoscience and Remote Sensing, vol. 52, no. 6, pp. 3502-3512, 2014. 